\title{
Single-Mode Beam-Cavity Interaction In Relativistic Klystrons*
}

\author{
S.M. Lidia, Lawrence Berkeley National Laboratory, Berkeley, CA 94720 USA
}

\section{Abstract}

The interaction between a modulated, intense electron beam and a single-mode rf cavity is discussed. A formalism is described which accounts for steady-state and transient beam loading, input/output waveguide coupling, and finite $\mathrm{Q}$ effects. A circuit equation is analyzed for shorttime behavior. Algorithms are presented for designing detuned structures to ensure longitudinal stability in relativistic klystron two-beam accelerators.

\section{FUNDAMENTAL ELEMENTS AND DYNAMICS}

We are specifically concerned with the interaction of the beam with the fundamental monopole mode $\left(T M_{010}\right)$ in a single standing-wave (SW) idler or output cavity. We express the cavity electric field as a product of a timedependent mode amplitude with a spatial mode profile (indexed by $\lambda$ ),

$$
\vec{E}(\vec{r}, t)=a_{\lambda}(t) \overrightarrow{E_{\lambda}}(\vec{r}) .
$$

The spatial profile of the mode is assumed to have the socalled 'Slater' normalization,

$$
\int_{\text {cavity }} d^{3} r \vec{E}_{\lambda}(\vec{r}) \cdot \vec{E}_{\lambda}(\vec{r})=1 .
$$

The other dynamical quantity is the current density representing the beam travelling through the cavity structure. We define a modal current density, $J_{\lambda}(t)$, by computing the overlap of the time-dependent current density with the spatial profile of the mode electric field, as in (2).

We may write down an equivalent circuit equation describing the time evolution of the mode amplitude due to excitation by both the external rf current drive and the incoming waveguide mode, and losses from wall heating, beam loading, and coupling to the outgoing waveguide mode [1] [2],

$$
\begin{gathered}
\left(\frac{d^{2}}{d t^{2}}+\frac{\omega_{\lambda}}{Q_{w}} \frac{d}{d t}+\omega_{\lambda}^{2}\right) a_{\lambda}(t)= \\
-\frac{1}{\epsilon_{0}} \frac{d}{d t} J_{\lambda}(t)+\frac{\omega_{\lambda}}{Q_{e x t}} \frac{d}{d t}\left(\frac{V_{1}^{+}-V_{1}^{-}}{V_{1 \lambda}}\right) .
\end{gathered}
$$

For quasi-steady-state harmonic oscillation at the modulation $\mathrm{rf}$ frequency, we express the time-dependence of the rf amplitudes as $a_{\lambda}(t) \cong \widehat{a_{\lambda}} \cos \left(\omega_{b} t+\varphi_{\lambda}\right), J_{\lambda}(t) \cong$ $\widehat{J_{\lambda}} \cos \left(\omega_{b} t\right), V_{1}^{ \pm}(t) \cong \widehat{v}^{ \pm} \cos \left(\omega_{b} t+\varphi_{ \pm}\right)$.

${ }^{*}$ This work supported under the auspices of the U.S. Department of Energy by LBNL under Contract No. AC03-76SF00098.

\subsection{Normalization of the Cavity Electric Field}

We only consider the on-axis longitudinal electric field profile and assume that it is only a function of longitudinal position $(z)$, with a separable time dependence. The values of the field and its derivatives along the axis are used to generate all other electric and magnetic field components (permitted by symmetry) near the axis by construction of the vector potential.

Without the detailed description of the total electric field profile throughout the entire cavity, we are unable to normalize the modes according to the Slater prescription (2). However, this normalization may be done when the modes are initially generated by electromagnetic codes [Superfish, URMEL, MAFIA, GdfidL, et. al.]. This can be performed through a combination of analytical modeling of the onaxis field profile with numerical calculation of the [R/Q]. This relates the on-axis voltage seen by the beam to the total energy stored in the cavity, and introduces a normalization constant, $N_{\lambda}$. In terms of the modal fields, the $a c$ celerator $[\mathrm{R} / \mathrm{Q}]$ is

$$
\left[\frac{R}{Q}\right]_{\lambda}=\frac{2}{\epsilon_{0} \omega_{\lambda}}\left|\int_{\text {cavity }} d z E_{z \lambda}(z) \exp \left[i \omega_{\lambda} z / \beta_{z} c\right]\right|^{2} .
$$

For a Gaussian distributed on-axis longitudinal electric field component, with RMS width $\sigma$ in $z$, the normalization constant is

$$
N_{\lambda}=\left(\frac{1}{2} \epsilon_{0} \omega_{\lambda}\left[\frac{R}{Q}\right]_{\lambda}\right)^{1 / 2} \exp \left[\frac{\omega_{\lambda}^{2} \sigma^{2}}{2 \beta_{z}^{2} c^{2}}\right] .
$$

This defines the connection between the modal fields used in the circuit analogy, and the fields used in determining the beam dynamics. We refer to this definition of the field as the 'line voltage' normalization.

\subsection{Periodic Klimontovich Current Distribution}

The connection between the discrete particle description employed by the tracking code and the current density used in the circuit equation is made by appealing to the Klimontovich distribution.

Since the amplitude of the modulated current density varies only very slowly on the rf time scale, the charge per bunch and the distribution function appear to be periodic when observed over a few rf periods' duration. We describe the time dependence of the charge density distribution by expanding in a Fourier series basis defined over a 
single rf period. The relativistic nature of the beam allows us to neglect the transverse current density components.

The modal current density at time $t_{0}$ is calculated to be

$$
\begin{gathered}
J_{\lambda}\left(t_{0}\right)=I_{b}\left\langle\int_{\text {cavity }} d z E_{z \lambda}\left(\overrightarrow{r_{\perp}}, z\right)\right\rangle+ \\
+2 I_{b} \sum_{l=1}^{\infty}\left\langle\int_{\text {cavity }} d z E_{z \lambda}\left(\overrightarrow{r_{\perp}}, z\right) \cos \left[l \omega_{b}\left(t_{0}-t(z)\right)\right]\right\rangle
\end{gathered}
$$

For the beams we consider here, only the $l=1$ term is necessary to retain for the monopole mode. Higher-order azimuthal modes may couple to higher harmonic components in the beam's spectrum.

We have denoted with angular brackets an average over the bunch distribution. Here, $t(z)$ is the arrival time at beamline position $z$ with transverse offset $\overrightarrow{r_{\perp}}$ of an element of the current density. It will be convenient to use the real parts of complex-valued quantities. We will define,

$$
\widetilde{w}\left(\omega, \overrightarrow{r_{\perp}}\right)=\int_{\text {cavity }} d z E_{z \lambda}\left(\overrightarrow{r_{\perp n}}, z\right) \exp [-i \omega t(z)] .
$$

The particular function defined by (6) plays an extremely important role in the dynamics of the beam and the evolution of the cavity field amplitude. It serves to define the cavity voltage, and hence the mode [R/Q] and shunt impedance. It contains transit time effects, and when applied to beam particle trajectories in the presence of a background rf field, it will compute beam loading contributions to the shunt impedance and the net energy deposited into the mode. We will refer to it as the mode transit function.

\section{ANALYSIS OF THE CIRCUIT EQUATION}

Two distinct time-scales of interest exist in these problems. A fast time-scale, where variations occur within an $\mathrm{rf}$ period, and a slow time-scale. The latter can be the fill time of the cavity $\left(T_{F}\right)$, the rise time of the driving current, or some other relevant time-scale. The mode amplitude and current density are modulated at the fast time-scale. But the evolution of the amplitude as well as any phase drift occurs on the slow time-scale. As a result, we may re-write the governing circuit equation in terms of these slowly-varying quantities and the slow time-scale.

\subsection{Slow Time-Scale Equation of Motion}

Introducing slow time variations into the modal amplitudes and phases: $\widehat{a_{\lambda}}=\widehat{a_{\lambda}}(t), \varphi_{\lambda}=\varphi_{\lambda}(t), \widehat{J_{\lambda}}=2 I_{b}(t)\left\langle\widetilde{w}^{\dagger}\right\rangle$, $\widehat{v^{ \pm}}=\widehat{v^{ \pm}}(t), \varphi_{ \pm}=\varphi_{ \pm}(t)$. These functions are required to be slowly varying in time with respect to the rf period. Substituting these into the circuit equation, and neglect second-order time derivatives of slowly-varying quantities to obtain,

$$
\begin{gathered}
\left(\omega_{\lambda}^{2}-\omega^{2}-\frac{i \omega \omega_{\lambda}}{Q_{w}}\right)\left(\widehat{a_{\lambda}} e^{-i \varphi_{\lambda}}\right)+ \\
\left(\frac{\omega_{\lambda}}{Q_{w}}-2 i \omega\right) \frac{d}{d t}\left(\widehat{a_{\lambda}} e^{-i \varphi_{\lambda}}\right)=
\end{gathered}
$$

$$
\begin{gathered}
=\frac{\omega_{\lambda}}{Q_{e x t} V_{1 \lambda}}\left(\frac{d}{d t}-i \omega\right)\left(\hat{v}^{+} e^{-i \varphi_{+}}-\widehat{v}^{-} e^{-i \varphi_{-}}\right) \\
-2 \frac{\left\langle\widetilde{w}^{\dagger}\right\rangle}{\epsilon_{0}}\left(\frac{d}{d t} I_{b}-i \omega I_{b}\right) .
\end{gathered}
$$

This equation can be simplified by introducing a voltage normalization. We define an on-axis cavity circuit voltage, and forward and reverse port voltages via $\tilde{V}_{c}=\left(\widetilde{w_{0}} \widehat{a}_{\lambda} e^{-i \varphi_{\lambda}}\right), \widetilde{V_{F}}=\left(\frac{\widetilde{w_{0}} \hat{v}_{e}{ }^{-i \varphi}+}{V_{1 \lambda}}\right)$, and $\widetilde{V_{R}}=$ $\left(\frac{\widetilde{w}_{0} \hat{v}^{-} e^{-i \varphi}-}{V_{1 \lambda}}\right)$, where $\widetilde{w_{0}}=\widetilde{w}\left(\overrightarrow{r_{\perp}}=0, \omega_{b}\right)$. As defined, these voltages are complex-valued. The bunch-averaged accelerator shunt impedance is defined through $R_{b}=$ $Q_{\lambda}\langle[R / Q]\rangle$, where

$$
\left\langle\left[\frac{R}{Q}\right]\right\rangle=\frac{2 \widetilde{w_{0}}\left\langle\widetilde{w}^{\dagger}\right\rangle}{\epsilon_{0} \omega_{\lambda}}
$$

The shunt impedance as defined here is a complexvalued quantity, but that it can be expressed as, $\left\langle\left[\frac{R}{Q}\right]\right\rangle=$ $\left[\frac{R}{Q}\right]_{\lambda} F_{b}$, where $\left[\frac{R}{Q}\right]_{\lambda}=2 \frac{\left|\tilde{w}_{0}\right|^{2}}{\epsilon_{0} \omega_{\lambda}}$ is a manifestly real-valued quantity, and $F_{b}$ is a complex-valued bunch distributiondependent form-factor, with magnitude of order unity. The shunt impedance is given by $R_{\lambda}=Q_{L}\left[\frac{R}{Q}\right]_{\lambda}$.

Using the continuity condition [2], we may express the reverse voltage in terms of the cavity and forward voltages, $\widetilde{V_{R}}=\widetilde{V}_{c}-\widetilde{V_{F}}$. From standard microwave terminology, we recall the definitions of the tuning angle, $\psi(\tan \psi=$ $\left.Q_{L}\left(\frac{\omega_{\lambda}}{\omega}-\frac{\omega}{\omega_{\lambda}}\right)\right)$, cavity fill-time, $T_{F}=\frac{2 Q_{L}}{\omega_{\lambda}}$, and coupling parameter, $\beta=\frac{Q_{\text {wall }}}{Q_{e x t}}$. We introduce the phase change in a fill time, $\nu=\omega T_{F}$, and $\alpha=(1+i \tan \psi)$. Finally, we re-scale the time dependence by the fill time, $\tau=t / T_{F}$, to obtain

$$
\begin{aligned}
\widetilde{V}_{c}^{\prime}+\alpha \widetilde{V_{e}}= & \frac{2 \beta}{\beta+1} \widetilde{V_{F}}\left(1+\frac{i}{\nu} \widetilde{V_{F}} / \widetilde{V_{F}}\right) \\
& -R_{\lambda} F_{b} I_{b}\left(1+\frac{i}{\nu} I_{b}^{\prime} / I_{b}\right),
\end{aligned}
$$

This is the main result of this section. We do not attempt to find a global solution over time, which requires inclusion of the self-consistent interaction of the cavity back upon the beam. Rather, we will seek a local solution, valid only over a short time duration (though still long compared to the fast time scale), as an approximation to use within the numerical simulation. We will take the form factor, $F_{b}$, as approximately constant over the interval.

\subsection{Observed Fields and Power Flow}

Of interest is the amount of rf power flowing into and out of the cavity. This power flow is derived from the Poynting flux. The net rms power flowing in the waveguide, again assuming a single mode, can then be shown to be

$$
\bar{P}_{+}=\frac{\epsilon_{0} \omega_{\lambda}}{2 Q_{e x t}}\left|\frac{\widetilde{V_{F}}}{\widehat{w_{0}}}\right|^{2}, \bar{P}_{-}=\frac{\epsilon_{0} \omega_{\lambda}}{2 Q_{e x t}}\left|\frac{\widetilde{V}_{e}-\widetilde{V_{F}}}{\widetilde{w_{0}}}\right|^{2}
$$

denoting the forward and reverse rms power flows in the connecting waveguide, respectively. Note that the reverse 
voltage has been expressed in terms of the forward and cavity voltages.

\section{INTERNAL BUNCH DYNAMICS AND THE AVERAGED SHUNT IMPEDANCE}

In the usual linac formulation, the function $\widetilde{w}_{0}$ is calculated by assuming a constant velocity, $\beta_{0}$, of particles through the cavity. In that case $t(z)=t_{0}+\left(z-z_{0}\right) /\left(\beta_{0} c\right)$. Here, we allow for intra-bunch particle motion resulting from a finite beam energy spread, and from the influence of rf fields generated from the interaction of previous bunches with the cavity. We determine the value of $t$ from the fiducial orbit.

For a SW monopole mode, we use

$$
E_{z \lambda}\left(\overrightarrow{r_{\perp}}, z\right)=f_{0}(z)+r_{\perp}^{2} f_{2}(z)+r_{\perp}^{4} f_{4}(z)+\cdots
$$

and the auxiliary functions are (primes denoting total derivatives with respect to $\mathrm{z}$ )

$$
\begin{aligned}
& f_{0}(z)=E_{z \lambda}\left(\overrightarrow{r_{\perp}}=0, z\right)=N_{\lambda} e_{0}(z) \\
& f_{2}(z)=-1 / 4 N_{\lambda}\left(e_{0}^{\prime \prime}+k_{0}^{2} e_{0}\right) \\
& f_{4}(z)=1 / 64 N_{\lambda}\left(e_{0}^{(i v)}+2 k_{0}^{2} e_{0}^{\prime \prime}+k_{0}^{4} e_{0}\right)
\end{aligned}
$$

and $k_{0}=\omega_{\lambda} / c$. We re-write the mode transit functions as

$$
\begin{aligned}
\widetilde{w} \cong \int_{\text {cavity }} d z & \left(f_{0}(z)+r_{\perp}^{2} f_{2}(z)+r_{\perp}^{4} f_{4}(z)\right) \times \\
& \times \exp \left[-i \omega_{b} t(z)\right] .
\end{aligned}
$$

For the beams of interest to us for RK-TBA devices, the longitudinal phase space is characterized by bunches which subtend a significant fraction of the rf wavelength. The bunch-averaged shunt impedance is evaluated by taking averages over the spatial and phase coordinates of the particles in the bunch. This serves to define the bunch-averaged form factor, $F_{b}$.

\section{DESIGN OF IDLER AND OUTPUT CAVITIES}

The collection of idler and output cavities in an RK-TBA requires detailed design so that its rf properties match the demands of the device in terms of beam transport stability and output power production. In particular, the resonant frequency and loaded Q-value of a cavity determine the longitudinal impedance seen by the beam. This impedance is tuned inductively to counteract the (capacitive) effects of space charge debunching and loss of rf current carried by the beam.

\subsection{Steady-State Scaling}

In this section, we consider the steady-state behavior of the beam-cavity system. We neglect the energy spread of the bunch as it passes through the cavity, and further assume that the individual particle velocities remain constant (the 'linac' approximation). The arrival time at the center of the cavity of a given particle is given by $t(z)=t_{0}+(z-$ $\left.z_{0}\right) /\left(\beta_{0} c\right)+\hat{s} / c$. The distribution in arrival time $(\widehat{s})$ is taken to be gaussian. Following the phase convention of (6), we set $t_{0}=0$. The transverse distribution is also taken to be gaussian, and essentially static. We take the bunch distribution to be $g\left(\overrightarrow{r_{\perp}}, \hat{s}\right)=\frac{\exp \left(-\frac{\sigma_{\perp}^{2}}{2 \sigma_{r}^{2}}\right)}{2 \pi \sigma_{r}^{2}} \frac{\exp \left(-\frac{\hat{s}^{2}}{2 \sigma_{s}^{2}}\right)}{\sqrt{2 \pi} \sigma_{s}}$, so that $\widetilde{w}=\widetilde{w_{0}} \exp \left(-i k_{b} \widehat{s}\right)=N_{\lambda} \exp \left(-\frac{k_{b}^{2} \sigma^{2}}{2 \beta_{0}^{2}}\right) \exp \left(-i k_{b} \widehat{s}\right)$, where $k_{b}=\omega_{b} / c$. And, in this case, the bunch-averaged form factor is real-valued, $F_{b}=\exp \left(-\frac{k_{b}^{2} \sigma_{s}^{2}}{2}\right)$. We can find the steady-state power flow out of the beam and into the wall and into the reverse waveguide mode,

$$
\begin{aligned}
\bar{P}_{b} & =R_{\lambda} I_{b}^{2} \cos ^{2} \psi \exp \left(-k_{b}^{2} \sigma_{s}^{2}\right), \\
\bar{P}_{w} & =\frac{Q_{L}}{Q_{w}} R_{\lambda} I_{b}^{2} \cos ^{2} \psi \exp \left(-k_{b}^{2} \sigma_{s}^{2}\right), \\
\bar{P}_{-} & =\frac{Q_{L}}{Q_{e x t}} R_{\lambda} I_{b}^{2} \cos ^{2} \psi \exp \left(-k_{b}^{2} \sigma_{s}^{2}\right) .
\end{aligned}
$$

\subsection{Beam Energy Modulation}

The beam energy distribution undergoes modulation from this field. The modulation of beam energy $(\gamma)$ is then

$$
\triangle \gamma(\widehat{s})=-\frac{R_{\lambda}\left|I_{b}\right|}{m_{e} c^{2} /|e|} \exp \left(-\frac{k_{b}^{2} \sigma_{s}^{2}}{2}\right) \cos \psi \cos \left(\psi+k_{b} \widehat{s}\right) \text {. }
$$

The cavity interaction thus produces a correlation between arrival time and energy.

\subsection{Inductive Detuning for Beam Stability}

The energy modulation imposed upon the beam by its interaction with the cavity contains a sinusoidal variation with arrival time and a phase offset determined by the cavity tuning angle. The tuning angle contains two free parameters, $\omega_{b}$ and $Q_{L}$. For RK-TBA applications, these parameters are used to adjust the amount of energy loss experienced between the head and tail of the rf bunch. Or, equivalently, the average bunch energy loss and the longitudinal phasespace rotation of the bunch. The average energy loss is determined by the rf power required, while the bunch rotation degree of freedom is used to counterbalance the opposite rotation (and debunching effect) of self-fields. The average energy change for the bunch is then found to be $\overline{\Delta \gamma}=-\frac{\bar{P}_{b}}{V_{0}\left|I_{b}\right|}=-\frac{\left(Q_{e x t} / Q_{L}\right) \bar{P}_{-}}{V_{0}\left|I_{b}\right|}$, where $V_{0}=m c^{2} / e$ is the electronic rest mass in voltage units.

\section{REFERENCES}

[1] J.C. Slater. "Microwave Electronics". D. Van Nostrand Company, Inc., 1950.

[2] D.H. Whittum. Introduction to electrodynamics for microwave linear accelerators. In RF Engineering for Particle Accelerators. World Scientific, 1998. 